\title{
Prevalence of asymptomatic Clostridium difficile colonization in tertiary hospital patients in Australia
}

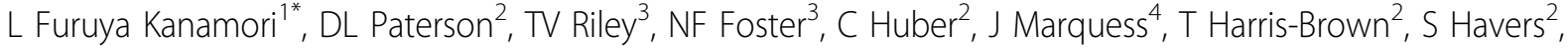 \\ SJ McKenzie ${ }^{5}, \mathrm{~L}$ Yakob $^{6}$, AC Clements ${ }^{1}$
}

From 3rd International Conference on Prevention and Infection Control (ICPIC 2015)

Geneva, Switzerland. 16-19 June 2015

\section{Introduction}

Despite the importance of $C$. difficile infection (CDI) as a cause of hospital-acquired diarrhea, few studies have investigated the prevalence of asymptomatic $C$. difficile colonization in a broad cross-section of the general hospital patient population over multiple years and seasons.

\section{Objectives}

To estimate the prevalence of asymptomatic $C$. difficile colonization of tertiary hospitals in different Australian States during six time-periods (late summer [Feb-Mar] and late winter [Aug-Sep]) 2012-2014 and to describe the diversity of PCR ribotypes isolated from asymptomatic patients.

\section{Methods}

A three-year repeated cross-sectional study with biannual surveys of randomly selected adult patients from all care wards in tertiary hospitals in Australia was conducted. Stool specimens were cultured for $C$. difficile and isolates were characterized by PCR ribotyping. Overall prevalence of asymptomatic $C$. difficile colonization, hospital and time-period specific prevalences were calculated and compared using logistic regression.

\section{Results}

Asymptomatic $C$. difficile colonization was identified in $112 / 1417$ (7.90\%; 95\% CI 6.55-9.43) patients during the study period. Asymptomatic C. difficile colonization prevalence was at its highest in Feb-Mar 2012 (11.95\%; 95\% CI 8.46-16.22), whereas the lowest prevalence was

${ }^{1}$ Research School of Population Health, The Australian National University, Canberra, Australia

Full list of author information is available at the end of the article observed in Aug-Sep 2014 (5.84\%; 95\% CI 3.30-9.44). A seasonal pattern characterized by lower prevalence in late winter (OR 0.63; 95\% CI 0.42-0.94) was identified. The majority of the isolates $(77.55 \%)$ were toxigenic C. difficile strains, PCR 014 and 018 were the most frequent toxigenic strains isolated.

\section{Conclusion}

High variability of asymptomatic $C$. difficile colonization prevalence was observed across seasons. The majority of the asymptomatic $C$. difficile infected patients were colonized by toxigenic strains.

"The study was funded by a NHRMC project grant (APP1006243).

\section{Disclosure of interest}

None declared.

\section{Authors' details}

${ }^{1}$ Research School of Population Health, The Australian National University, Canberra, Australia. ${ }^{2}$ University of Queensland Centre for Clinical Research, University of Queensland, Herston, Australia. ${ }^{3}$ School of Pathology and Laboratory Medicine, University of Western Australia, Perth, Australia. ${ }^{4}$ Centre for Healthcare Related Infection Surveillance and Prevention, Queensland Health, Queensland, Australia. ${ }^{5}$ School of Population Health, University of Queensland, Herston, Australia. ${ }^{6}$ Department of Disease Control, London School of Hygiene and Tropical Medicine, London, UK.

Published: 16 June 2015

doi:10.1186/2047-2994-4-S1-O33

Cite this article as: Kanamori et al:: Prevalence of asymptomatic

Clostridium difficile colonization in tertiary hospital patients in Australia. Antimicrobial Resistance and Infection Control 2015 4(Suppl 1):O33. 